\title{
A Preliminary Study on The Prevention and Control of Seawater Intrusion in Northern Pingtan Island with Freshwater Recharge Curtain
}

\author{
Mengyao Diao ${ }^{1, *}$, Panlin $\mathrm{Li}^{1}$, Weifang Ruan ${ }^{2}$, Wanglin $\mathrm{Li}^{1}$ \\ ${ }^{1}$ University of Jinan, Jinan 250022, China \\ ${ }^{2}$ Fujian Institute of Water Resources and Hydropower, Fuzhou 350001, China
}

\begin{abstract}
Seawater intrusion caused by groundwater over-exploitation is a kind of geological disaster caused by human economic activities and natural environment changes. Using freshwater recharge curtain technology is an effective means to prevent seawater intrusion. Based on the analysis of the characteristics of seawater intrusion in Pingtan island, Fujian Province, and in view of the possible seawater intrusion problems in the construction of Luyangpu underground reservoir in Pingtan Island, this paper expounds the basic principle of seawater intrusion prevention with freshwater recharge curtain, the technical measures of installing a row of recharge wells in the northern coastal zone of Luyangpu plain in Pingtan Island and using freshwater recharge curtain are put forward to prevent seawater intrusion. Through groundwater numerical simulation, the dynamic distribution of groundwater level in the north of Pingtan Island in the next 20 years is analyzed and predicted. The results show that the seawater intrusion can be effectively prevented by installing freshwater curtain recharge wells in the northern coast of Luyangpu.
\end{abstract}

\section{Introduction}

In recent years, seawater intrusion in coastal zone can not be ignored. As a geological disaster, it is seriously affecting the lives and economic development of local residents. Seawater intrusion is caused by over-exploitation of groundwater, and is affected by geology, lithologic and atmospheric precipitation and other factors ${ }^{[1]}$.

At home and abroad, the technical measures to prevent seawater intrusion include: strengthening the control of pumping wells in coastal areas, optimizing groundwater exploitation technology, hydraulic curtain (water injection curtain and water pump-injection curtain) and bubble curtain technology, underground dam, compressed gas injection technology in confined aquifer and comprehensive prevention and control technology. Among them, hydraulic curtain and underground dam are most often used in the practice of preventing seawater intrusion. Increasing groundwater recharge, properly retaining groundwater runoff to reduce the flux of underground freshwater into the sea, and constructing groundwater hydraulic curtain can intercept groundwater runoff and prevent seawater intrusion ${ }^{[1]}$. In the technical research of preventing seawater intrusion by hydraulic curtain, foreign countries started earlier, and the earliest research can be traced back to the 19th century. J.Du Commun (1828), Bado n-Ghyben (1889) and Herzberg (1901), gave the expression of the depth below sea level at any point on the interface between salt water and freshwater when seawater invaded ${ }^{[2]}$. In 1953, the United States carried out the artificial recharge project along the coast of Los Angeles, the water in the reservoir in the northern mountain area was used to recharge the groundwater and raise the groundwater level, which formed a freshwater barrier to effectively prevent

*Corresponding author: dmy_9898@163.com 
seawater intrusion ${ }^{[3]}$. In 1972, the Teboulba aquifer in Tunisia was recharged by rainwater collected from the dam, which made the sag disappear before recharging, improved the storage capacity of aquifer and effectively prevented seawater intrusion ${ }^{[4]}$. The prevention and control of seawater intrusion started relatively late in China, but the research on seawater intrusion theory developed rapidly, and now it is close to the international advanced level. In 2007, Liu Qingyong, Pan Shibing and $\mathrm{Wu}$ Xiaofeng have prevented the seawater intrusion through the technology of groundwater recharge in Guangrao County, Shandong Province ${ }^{[5]}$.A seawater intrusion prevention and control system invented by Wang Xiaojun and Chen Feng in 2018 solves the problem that the current technology can only prevent or desalinate seawater intrusion, realizes the combination of prevention and utilization, and improves the application efficiency of the system ${ }^{[6]}$. In the study of measures for recharging freshwater, Niu Sixiang changed the recharge rate of freshwater, which significantly shortened the distance of seawater intrusion. The larger the recharge amount of freshwater, the smaller the intrusion distance of seawater. The larger the recharge amount, the more effective to prevent seawater intrusion ${ }^{[7]}$.

In view of the possible seawater intrusion in Pingtan Island, this paper studies the technical measures to prevent seawater intrusion by using freshwater recharge curtain, and verifies the feasibility of the measures by numerical simulation analysis.

\section{Characteristics of intrusion in Pingtan Island}

\subsection{Geological conditions}

Pingtan Island is located in the eastern part of Fujian Province, with flat terrain, mainly marine plain, and Junshan is the highest peak of the whole island. There are Pre-Devonian Qinyingshan Formation, Late Jurassic Nanyuan Formation and Quaternary strata in Pingtan Island, among which Quaternary strata are well developed and can be divided into Holocene aeolian deposits, marine deposits, slope diluvial deposits and Pleistocene marine deposits, slope diluvial deposits and residual deposits. The typical geological profile of
Luyangpu in Pingtan Island is shown in Figure 1.

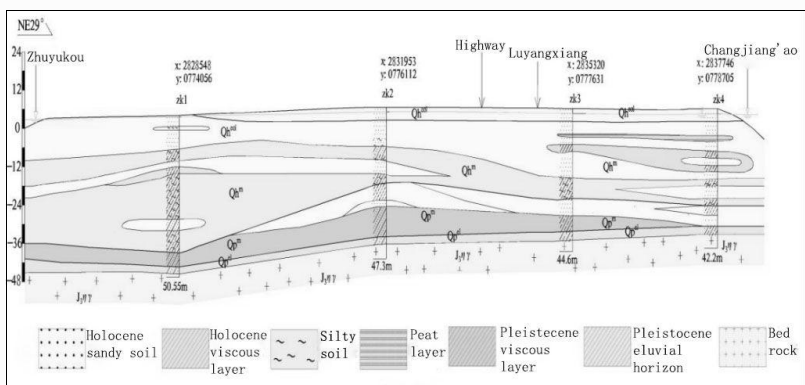

Fig.1. Geological profile of Luyangpu in Pingtan Island

The aquifer type of Pingtan Island is mainly pore aquifer, which is composed of Quaternary Holocene aeolian, marine fine sand, medium fine sand and fine sand, with uniform particles and good water permeability. The average aquifer thickness is $19.38 \mathrm{~m}$, the groundwater level depth is $0.70 \sim 1.42 \mathrm{~m}$, the common value of single well water inflow is $1002 \sim 1348 \mathrm{~m}^{3} / \mathrm{d}$, and the grade of water abundance is abundant.

Atmospheric precipitation is the main recharge of aquifer, followed by lateral recharge of bedrock fissure water. Pingtan Island is flat, with slow underground runoff and weak hydrological cycle. Groundwater is mainly evaporated by capillarity or discharged into the sea by an undercurrent. Bedrock fissure water is recharged by atmospheric precipitation, and is mostly discharged by surface runoff. Hydrological cycle is fast, and it is generally recharged and discharged locally. Groundwater is usually collected in the form of springs, supplied plain areas or flowed into the sea.

Groundwater dynamic changes obviously with climate in Pingtan Island. During the rainy season, the water level rises sharply; During the dry season, the water level drops slowly. The period of high groundwater level is from mid-February to September, and the period of low groundwater level in dry season is from October to February of the following year.

\subsection{Characteristics of seawater intrusion}

In the natural state, the salt water and freshwater in the coastal underground aquifer is in a state of dynamic equilibrium. Because of over-exploitation of groundwater, the water level of freshwater drops, the equilibrium interface between salt water and fresh water is destroyed, and the seawater intrudes into the 
freshwater aquifer, resulting in salty water quality ${ }^{[8]}$.

Seawater intrusion is serious in some areas of Pingtan Island, the salt and fresh water areas are mainly distributed in the central part of the western coast of Pingtan Island, mainly due to the over-exploitation of groundwater, high-level mariculture and the overall sea level rise caused by global warming.

Seawater intrusion affects people's lives, endangers people's health, affects industrial production, and also leads to land salinization, which seriously restricts the economic development of Pingtan Island.

An underground reservoir is planned to be built in the north of Luyangpu in Pingtan Island. If the underground reservoir is not developed properly and effective measures for groundwater protection are lacking, the groundwater will continue to drop sharply, forming a certain cone of depression, and inducing new seawater intrusion, which will have a serious impact on the development of Pingtan Island.

\section{Prevention and control technology of seawater intrusion by freshwater recharge curtain in Pingtan Island}

\subsection{Basic principle of preventing seawater intrusion with freshwater curtain}

The following illustrates the basic principle of seawater intrusion prevention with freshwater recharge curtain through single well recharge curtain, and the schematic diagram is shown in Figure 2. When a recharge well is arranged on the onshore freshwater side of the coastline, a recharge mound is formed between freshwater and seawater on the section perpendicular to the coastline, which can block seawater from invading freshwater.

A row of recharge wells is install on the freshwater side of the land parallel to the coastline. By pumping freshwater from the reservoir area, the recharge wells are used to recharge groundwater, thus forming a plurality of recharge hills. When the well spacing of single recharge wells is less than the influence radius of recharge wells, the recharge wells interfere with each other, which can form a continuous reinjection mound, that is, freshwater reinjection curtain. By controlling the well spacing of recharge wells, the water level in the overlapping part of recharge mounds of two adjacent recharge wells can be higher than the sea level, thus forming a barrier between inland freshwater and seawater, blocking seawater from invading underground freshwater aquifers and preventing seawater intrusion. In addition, the freshwater curtain can effectively block underground freshwater from flowing into the sea.

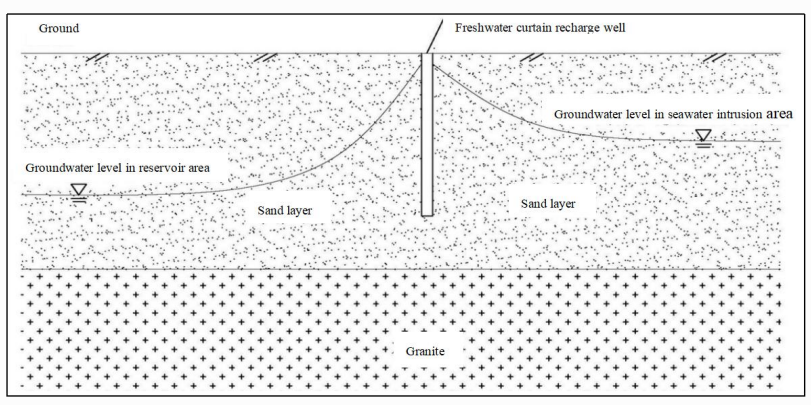

Fig.2. Schematic diagram of the principle of freshwater recharge curtain

The formation of freshwater recharge curtain is closely related to freshwater recharge amount, salt water concentration, aquifer thickness and characteristics of porous media. The formation process of freshwater recharge curtain includes formation stage, development stage and seawater intrusion stage. In the formation stage, under the condition of recharging freshwater, the freshwater curtain develops horizontally and vertically at the same time, and the development speed increases from the beginning, and then decreased, finally tends to be flat; In the development stage, when the freshwater curtain develops to the bottom of the aquifer in the vertical direction, it forms a freshwater curtain, and the relationship between the development speed and the freshwater quantity and salt water concentration shows a good regularity. In the seawater intrusion stage, when the recharge of freshwater is stopped, salt water will gradually invade inland ${ }^{[9]}$.Liu Libo and others studied the prevention of seawater intrusion by freshwater recharge curtain through numerical simulation. The results show that freshwater recharge flow plays a very significant role in the formation of freshwater curtain, and the greater the recharge amount, the faster the formation of freshwater curtain ${ }^{[10]}$. 


\subsection{Prevention and control scheme of seawater intrusion in Luyangpu, Pingtan}

Luyangpu underground reservoir is located in the north of Pingtan Island, and the northern boundary of the reservoir area is close to the coastal zone of Changjiang'ao. During the operation of the underground reservoir, when the groundwater level in the reservoir area is lower than the seawater level, seawater will often be poured into the underground aquifer in the reservoir area.

In order to avoid seawater from intruding into underground aquifers, a row of recharge wells can be arranged on the back side of the Changjiang'ao tide barrier in the north of Pingtan Island. By extracting freshwater from the aquifer in the reservoir area or collecting rain flood and using recharge well to recharge the aquifer, groundwater freshwater recharge curtain is formed, which can not only block the underground current of reservoir area flowing into the sea, but also prevent seawater from invading Luyangpu Plain in the Changjiang'ao direction. The layout of curtain recharge wells for freshwater recharge is shown in Figure 3.

Groundwater recharge sources of Luyangpu underground reservoir in Pingtan Island are mainly rainfall infiltration in the reservoir area, and slope runoff, rain flood collected from Jun Mountain and Longtou Mountain on both sides of the reservoir area.

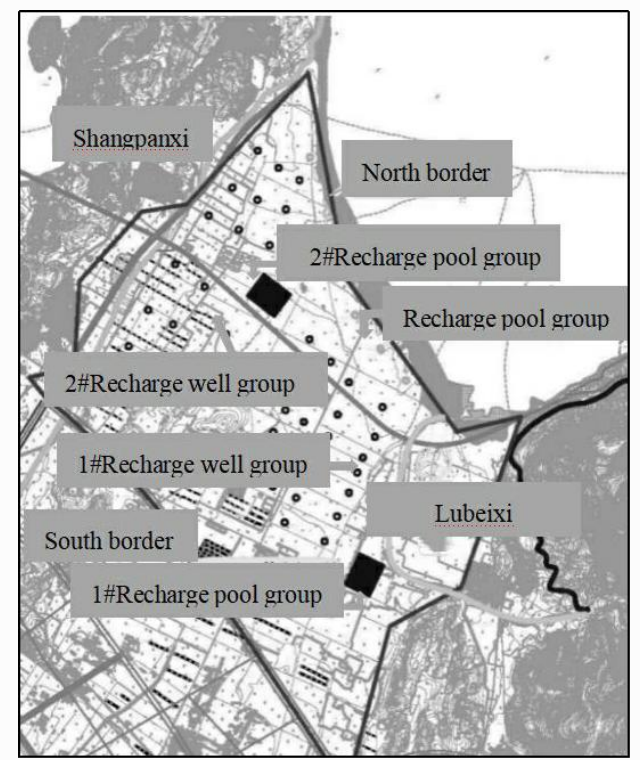

Fig.3. Layout of curtain recharge wells for freshwater recharge

\subsection{Demonstration of seawater intrusion prevention scheme with freshwater recharge curtain}

The groundwater mathematical model of Luyangpu Aquifer was established, and the dynamic change process of groundwater level with time at curtain recharge site at the northern boundary of the reservoir area was analyzed by Visual MODFLOW to verify the effectiveness of freshwater recharge curtain in preventing seawater intrusion.

\subsubsection{Hydrogeological model}

The whole Luyangpu Plain between Longtou Mountain and Jun Mountain is selected as the groundwater simulation area, including all Luyang Township, Zhonglou Township and Pingyuan Town, as well as part of Lancheng township, with a total simulation area of $75.1 \mathrm{~km}^{2}$.

Groundwater in the reservoir area is closely related to the upper and lower levels, which is characterized by phreatic water, and can be generalized as a heterogeneous isotropic three-dimensional phreatic water model in calculation. The lithology of underground aquifer is mainly Holocene medium fine sand and Pleistocene medium sand.

The eastern boundary of the reservoir area is Jun Mountain and the western boundary is Longtou Mountain, both of which are impervious boundaries. The northern border is the coastal zone of the Changjiang'ao, and it is planned to build a freshwater recharge curtain to block the intrusion of salt water into the aquifer. The southern boundary is the central plain of Luyangpu, which belongs to the permeable supply boundary and seawater intrusion is not considered.

In order to more intuitively observe the change trend of coastal water level in Pingtan Island under the freshwater curtain scheme, six underground water level observation wells are arranged along the freshwater curtain belt, and their positions are shown in Figure 4 . 


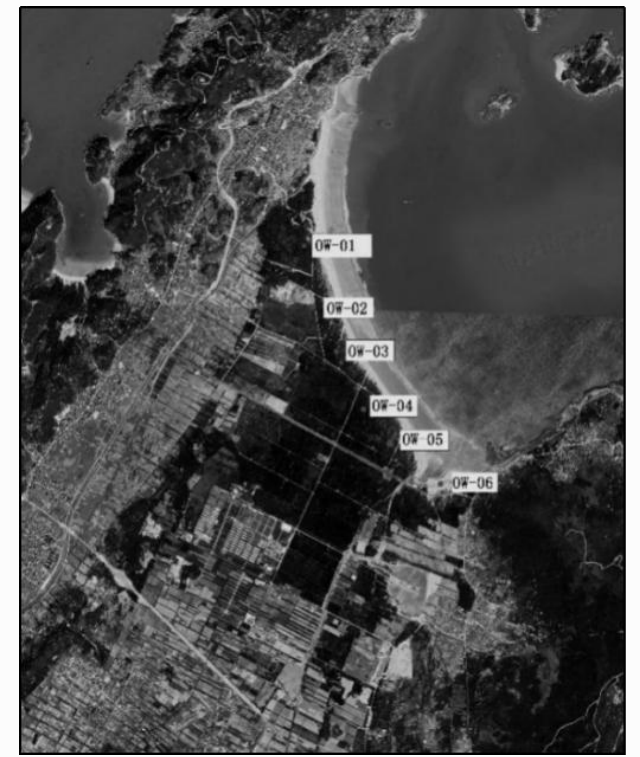

Fig.4. Simulation range and observation well layout of Luyangpu underground reservoir

\subsubsection{Establish a mathematical model}

Mathematical formula and definite solution condition of groundwater:

\subsubsection{Source and sink items}

Source and sink items of groundwater system include recharge items and discharge items. In this simulation area, the recharge items include atmospheric precipitation and artificial recharge, with an average of about 410 million $\mathrm{m}^{2}$ and $5,762,800 \mathrm{~m}^{2}$ every year respectively. Because the groundwater at the impermeable boundary is fissure water with very little content, lateral recharge in front of the mountain on both sides of the reservoir area is not considered. The discharge items are mainly artificial exploitation, which is determined by local data.

\subsubsection{Identification and Verification of Models}

The model parameters are modified and adjusted by trial estimation-correction method. The observation value of underground reservoir on November 24, 2015 is selected as the initial observation value of model identification, and the observation water level value of underground

$$
\begin{cases}\frac{\partial}{\partial x}\left(K_{x} \frac{\partial H}{\partial x}\right)+\frac{\partial}{\partial y}\left(K_{y} \frac{\partial H}{\partial y}\right)+\frac{\partial}{\partial z}\left(K_{z} \frac{\partial H}{\partial z}\right)+W=S \frac{\partial H}{\partial t} & (x, y, z) \in D \\ K_{x}\left(\frac{\partial H}{\partial x}\right)^{2}+K_{y}\left(\frac{\partial H}{\partial y}\right)^{2}+K_{y}\left(\frac{\partial H}{\partial y}\right)^{2}-\frac{\partial H}{\partial z}\left(K_{z}+p\right)+p=\mu \frac{\partial H}{\partial t} & (x, y, z) \in \Gamma_{0} \\ \left.H(x, y, z)\right|_{t=0}=H_{0}(x, y, z) & (x, y, z) \in D \\ \left.H(x, y, z, t)\right|_{\Gamma_{1}}=H_{1}(x, y, z, t) & (x, y, z) \in \Gamma_{1}, t>0 \\ \left.K_{n} \frac{\partial H}{\partial n}\right|_{\Gamma_{2}}=q(x, y, z, t) & (x, y, z) \in \Gamma_{2}, t>0\end{cases}
$$

In the formula, $\mathrm{D}$ is seepage area and $\mathrm{H}$ is groundwater head, $\mathrm{K}_{\mathrm{x}}, \mathrm{K}_{\mathrm{y}}, \mathrm{K}_{\mathrm{z}}$ are the permeability coefficient in $\mathrm{x}, \mathrm{y}$ and $\mathrm{z}$ directions, $\mathrm{S}$ is the unit water storage coefficient of aquifer below the free water surface, $w$ is the source and sink term of aquifer, $p$ is the evaporation and precipitation recharge of diving surface, $\mu$ is aquifer gravity specific yield, $H_{0}(x, y, z)$ is initial flow field head distribution value, $H_{1}(x, y, z, t)$ is the first kind of boundary head distribution value, $q(x, y, z, t)$ is the second kind of boundary flow per unit area, $K_{n}$ is permeability coefficient of boundary surface in outer line direction, $\Gamma_{1}$ is the first boundary of seepage area, $\Gamma_{2}$ is the second boundary of seepage area. reservoir from November 24, 2015 to October 2, 2016 is selected as the verification stage of this model research. It is verified that the number of observation wells with water level difference less than $0.5 \mathrm{~m}$ accounts for $80 \%$ of the total observation wells, and the simulation fitting degree is high, which meets the requirements.

\subsection{Result analysis}

The regulation and storage scheme of Luyangpu underground reservoir is numerically simulated, and the change of groundwater level in the next 20 years is predicted. The prediction curve of groundwater level of typical observation well (No.03) in the middle part of the 
freshwater recharge curtain in the next 20 years is shown in Figure 5, and the prediction curve of groundwater level along the freshwater recharge curtain in the next 20 years is shown in Figure 6.

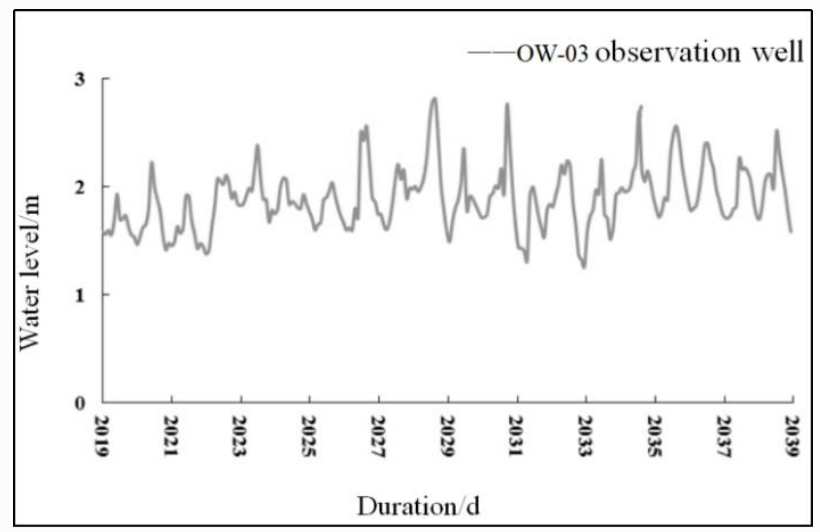

Fig.5. Groundwater level curves of typical observation wells in the next 20 years

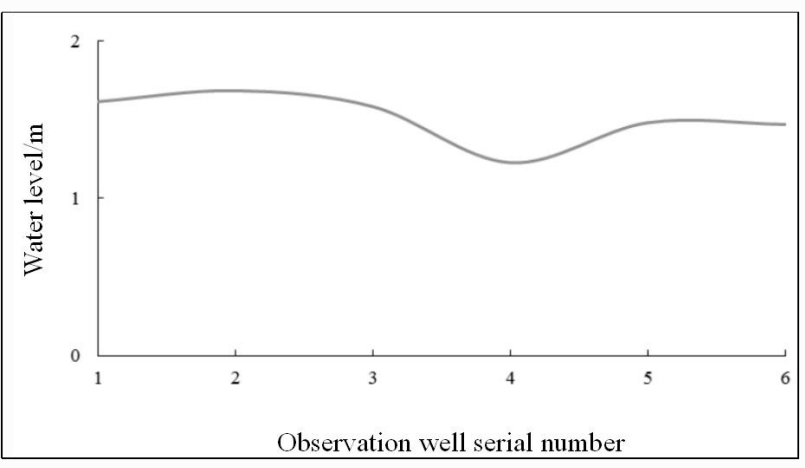

Fig.6. Prediction curve of groundwater level along freshwater recharge curtain in the next 20 years

It can be seen from Figure 5 and Figure 6 that during the operation of the underground reservoir in the next 20 years, the groundwater level along the freshwater recharge curtain belt and the typical groundwater level observation well (the middle position of the recharge curtain line) is higher than $0 \mathrm{~m}$, which indicates that the groundwater level in the north of the reservoir area near the Changjiang'ao is higher than the seawater level. Finally, the purpose of preventing seawater from flowing into freshwater aquifer is achieved, which means that the freshwater recharge curtain well can effectively control seawater intrusion.

\section{Conclusions}

Through the above analysis, the following conclusions are drawn:

(1) The main causes of seawater intrusion in
Pingtan Island are over-exploitation of groundwater, high-level mariculture and the overall rise of sea level caused by global warming.

(2) The principle of preventing seawater intrusion with freshwater recharge curtain is to set a row of recharge wells on the onshore freshwater side of coastline, and use freshwater recharge mound to form a barrier between inland freshwater and seawater, thus blocking seawater intrusion into underground aquifer.

(3) A row of recharge wells is set on the coastal side in the north of Luyangpu, Pingtan Island. Freshwater recharge is used to recharge the aquifer, forming a freshwater recharge curtain, which can effectively prevent seawater intrusion.

\section{References:}

1. Z.R. Guo, Y.P. Huang. Seawater Intrusion. 03, 10$15+9(2003)$

2. Z.H. Cai, F.S. Ma. Seawater intrusion and intrusi on development, 03, 1-9(1996)

3. X. Zhou, M.Y. Chen, X.M. Ju, J.P. Wang, X.S.

Ning. Seawater intrusion and prevention measures, 0

2, 78-84+92(1997)

4. Salem Bouri, Hamed Ben Dhia. Artificial recharge, 01, 342(2009)

5. Q.Y. Liu, S.B. Pan, X.F. Wu, W.H. Song. Ground water recharge technology,133-137(2007)

6. X.J. Wang, F. Chen. Seawater intrusion prevention, (2019)

7. S.X. Niu. Seawater intrusion, (2017)

8. M.G. You. Geologic environmental background, 05, 666-668(2009)

9. G.S. Zhang. Seawater intrusion, (2007)

10. L.B. Liu, G.S. Zhang, A.N. Jiang. Freshwater cu rtain, S1, 8-10(2007) 